17. Horz, W. \& Altenburger, W. Nucleic Acids Res. 9, 2643-2658 (1981).

18. Weiner, A., Hughes, A., Yassour, M., Rando, O.J. \&
(2008).

21. Fedor, M.J., Lue, N.F. \& Kornberg, R.D. J. Mol. Biol. 204, 109-127 (1988).

\title{
A preoccupied position on nucleosomes
}

\section{To the Editor:}

'Occupancy' is a measure of histone or nucleosome density. Occupancy is typically measured on a genomic scale using microarrays or through deep sequencing (Fig. 1). Kaplan et al. ${ }^{1}$ were correct in that the underlying DNA sequence has a predominant influence on occupancy levels in vivo. However, this and related work ${ }^{1-3}$ often interchanged the term 'occupancy' with 'positioning', which is confusing. 'Positioning' is a measure of the extent to which a population of nucleosomes resists deviating from its consensus location along the DNA and can be thought of in terms of a single reference point on the nucleosome, like its dyad (Fig. 1) ${ }^{4}$. A low standard deviation means high positioning. Zhang et al. ${ }^{5,6}$ were correct in that the underlying DNA sequences are not widespread determinants of nucleosome positioning in vivo, although they are major determinants at some positions. An important question now is how nucleosomes become uniformly spaced and precisely positioned in vivo.

\section{COMPETING FINANCIAL INTERESTS}

The author declares no competing financial interests.

\section{B Franklin Pugh}

Department of Biochemistry and Molecular Biology, The Pennsylvania State University, University Park, Pennsylvania, USA. e-mail:bfp2@psu.edu

1. Kaplan, N. et al. Nature 458, 362-366 (2009).

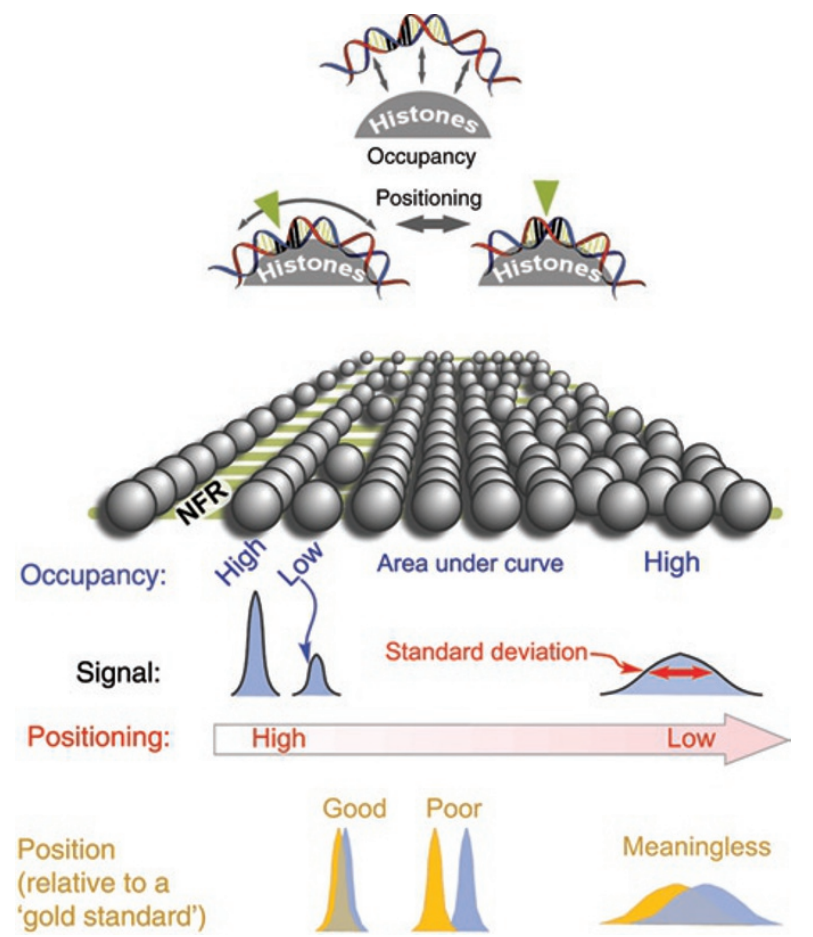

Figure 1 Illustration of how nucleosome occupancy and positioning differ. The upper panel shows a cross-section of a nucleosome, in which occupancy is distinguished from positioning. The lower panel shows how the two are measured. Occupancy is the area under the curve and reflects the local density of nucleosomes in a population, as illustrated by the column of spheres. Positioning or fuzziness is reflected in the standard deviation of the curve and is illustrated by how well the spheres are aligned in a column. The position of a nucleosome relative to some standard is indicated by how closely two peaks are separated. Comparing peaks of curves having high standard deviations is not likely to be meaningful because both peak locations have very high uncertainty.
2. Segal, E. et al. Nature 442, 772-778 (2006).

3. Kaplan, N. et al. Nat. Struct. Mol. Biol. 17, 918-923 (2010).

4. Albert, I. et al. Nature 446, 572-576 (2007)
5. Zhang, Y. et al. Nat. Struct. Mol. Biol. 16, 847-852 (2009).

6. Zhang, Y. et al. Nat. Struct. Mol. Biol. 17, 920-923 (2010) 